\title{
Preoperative graft assessment in aortocoronary bypass surgery
}

\author{
GRIGORE TINICA ${ }^{1,2}$, CRISTINA LUCA VARTIC ${ }^{1,2}$, VERONICA MOCANU ${ }^{2}$, \\ DANA BARAN $^{2}$ and DOINA BUTCOVAN ${ }^{1,2}$
}

\author{
${ }^{1}$ Department of Cardiovascular Surgery, 'Prof. Dr. George I.M. Georgescu' Institute of Cardiovascular Diseases, Iasi 700503; \\ ${ }^{2}$ Department of Morpho-Functional Sciences, 'Grigore T. Popa' University of Medicine and Pharmacy, Iasi 700115, Romania
}

Received November 17, 2014; Accepted December 16, 2015

DOI: $10.3892 / \mathrm{etm} .2016 .3412$

\begin{abstract}
Coronary artery bypass graft (CABG) is a surgical procedure able to improve the blood supply to the myocardium. In the present study, the distal segments of grafts taken from the internal thoracic artery (ITA), radial artery (RA) and saphenous vein (SV) for use in aortocoronary bypass surgery were examined. The morphologies of the grafts were investigated in order to draw conclusions concerning their patency and viability. In addition, clinical and laboratory risk factors considered to be significant predictors of lesion severity in graft vessels used in CABGs were investigated. In total, 54 distal graft segments of ITAs, RAs and SVs from 20 men and 6 women aged between 42 and 78 years, were evaluated. Histological analyses were used to visualize graft lesions. Morphometrically, the intimal thickness index (ITI) and luminal narrowing were assessed as an indication of graft patency. The histological changes observed in the graft vessel walls included the presence of distinct atheromatous plaques (fatty streaks in 2 cases) or thickening of the intima (20 cases) and media (17 cases). Morphometric analysis showed that the mean ITI of the vessel conduits was 0.37 in the SVs, 0.95 in the RAs, and 1.66 in the ITAs. No patient had $>50 \%$ conduit stenosis. By assessing the association between risk factors and graft lesions, it was found that all the patients showed risk factors for atherosclerosis, such as age (61.54\%), arterial hypertension (65.38\%), hyperlipidemia (65.38\%), smoking (34.61\%), diabetes mellitus (38.46\%) and obesity (15.38\%). The presence of pre-existing lesions in bypass grafts may contribute to a reduction in their viability, particularly in the case of venous grafts. Further long-term follow-ups are mandatory to evaluate the consequences of such lesions upon the patency of the grafts.
\end{abstract}

Correspondence to: Professor Veronica Mocanu, Department of Morpho-functional Sciences, 'Grigore T. Popa' University of Medicine and Pharmacy, 16 Universitatii Street, Iasi 700115, Romania

E-mail: vmocanu@iasi.mednet.ro

Key words: aortocoronary bypass, graft, internal thoracic artery, radial artery, saphenous vein

\section{Introduction}

Coronary artery bypass graft (CABG) surgery is a procedure that can improve the supply of blood to the myocardium, and is conducted in order to relieve angina and decrease the risk of mortality from coronary artery disease $(1,2)$. The most commonly used conduits in CABG surgery are the saphenous vein (SV), internal thoracic artery (ITA) and radial artery (RA) (3). The choice and quality of the conduits used for revascularization have a major role in the success of CABG, with the long-term patency of these conduits being particularly important. The SV was the first used conduit in CABG surgery, and remains the most common, because of its relatively large diameter and technical ease of use. However, the ITA is currently the first choice of bypass conduits due to its excellent results, with a patency rate of $90 \%$ at 10 years after CABG. The RA is often considered as the second graft of choice after the ITA, having a high patency rates close to $90 \%$ up to 10 years after CABG (4).

The CABG technique consists in taking arteries or veins from elsewhere in the patient's body and grafting them in order to bypass atherosclerotic narrowings (5). Usually a patient might receive between two and five bypass grafts, including arteries such as the ITA and RA, and veins, such as the SV $(6,7)$. The lack of a convenient method to objectively assess pre-operatively the intimal quality may lead to early graft failures $(8,9)$.

The aim of the present morphological and morphometric study was to conduct an analysis of grafts from the ITA, RA and SV, prior to their use in aortocoronary bypass surgery, in order to identify pre-operative histological changes and to draw conclusions concerning the viability of the grafts. Another aim was the assessment of clinical and laboratory risk factors (10) considered to be significant predictors of lesion severity in intimal hyperplasia, atherosclerosis and other pathologies treated by CABGs.

\section{Materials and methods}

Patient enrolment. A total of 26 patients who were undergoing surgical coronary revascularization at the 'Prof. Dr. George I.M. Georgescu' Institute of Cardiovascular Diseases (Iasi, Romania) were enrolled in the present study between January 2013 and December 2013. Their ages ranged from 42-78 years (mean age, 60 years). The patients comprised 20 men $(76.92 \%)$ and 6 women $(23.08 \%)$. Ethical approval 
for the experiments conducted in the present study was obtained from the Institutional Board of 'Prof. Dr. George I.M. Georgescu' Institute of Cardiovascular Diseases. Written informed consent was obtained from all patients.

Morphological and morphometric analysis of the grafts. A total of 54 distal segments of the ITA, RA and SV were evaluated. For histological examination, all sections were stained with hematoxylin and eosin (H\&E), as well as with elastic Van Gieson (EVG) and Sirius red (SR) stains. Histological assessment was made with an optical microscope (Olympus CX41; Olympus Corporation, Tokyo, Japan). The measurements were conducted using a color image analysis system (QuickPHOTO MICRO 3.0; Promicra, Prague, Czech Republic). Intima and media thickness and surface area were measured, in order to assess the intimal thickness index (ITI) and luminal narrowing.

Evaluation of the ITI and intimal narrowing. The degree of intimal thickening (by intimal hyperplasia and atherosclerosis) and luminal narrowing of the vascular conduits was evaluated by the determination of the ITI, which was calculated from the ratio of intimal and medial areas. The intima was defined as the distance from the lumen to the internal elastic lamella (IEL), in the area with the greatest intimal thickness. The media was considered as the distance from the internal elastic lamella to the adventitia, at the level of the greatest medial thickness. Two severity indices were calculated from the most severely diseased sections of the specimens using the following formulae: i) ITI = intimal area/medial area; and ii) luminal narrowing $(\%)=$ intimal area/IEL area $x 100$. The ratio of the thickness of the intima to that of the media (R) was used as the index for arteriosclerosis, in accordance with the method of Kobayashi et al (11). Atherosclerosis was graded on the basis of $\mathrm{R}$ as follows: Grade I, insignificant $(\mathrm{R}<0.1)$; grade II, mild $(0.1 \leq \mathrm{R}<1.0)$; grade III, moderate $(1.0 \leq \mathrm{R}<3.0)$; and grade IV, severe $(\mathrm{R} \geq 3.0)$.

Risk factor analysis. The prevalence of potential risk factors for atherosclerosis were assessed as follows: Age, diabetes mellitus (DM), arterial hypertension (AHT), history of cigarette smoking, hyperlipidemia and obesity. The associations between lesion severity and the number of atherosclerotic risk factors were investigated. Results are expressed as mean values or frequencies.

\section{Results}

Patient characteristics. All analyzed patients presented signs of unstable angina pectoris at hospital admission. Table I shows the vessels used for myocardial revascularization according to patient gender.

Morphological analysis. The results of the histological investigation (Table II) indicated that morphological changes were present with high incidence in the walls of the fresh 'normal' vessels (ITAs, RAs and SVs) prior to their use as aortocoronary conduits. The identification of the presence of preoperatory vessel lesions is very important in the viability assessment of the graft conduits and for long-term assessment. According
Table I. Vascular conduits according to gender.

\begin{tabular}{lrrrr}
\hline Gender & ITA & RA & SV & Total \\
\hline Male & 20 & 10 & 18 & 48 \\
Female & 4 & 2 & 0 & 6 \\
Total & 24 & 12 & 18 & 54 \\
\hline
\end{tabular}

ITA, internal thoracic artery; RA, radial artery; SV, saphenous vein.

to their descending order of frequency, the graft lesions were represented by intimal thickening or hyperplasia (20 cases), medial fibrosis (17 cases) and fatty streaks (2 cases).

Morphological examination indicated that the ITA lesions consisted mainly of intimal hyperplasia associated with intimal thickening (Fig. 1A), medial fibrosis (including 1 case with medial dissection) and fatty streaks (Fig. 1B). The RA lesions consisted of intimal thickening (Fig. 2A) and medial fibrosis, in equal proportions. SV lesions consisted mainly of intimal hyperplasia, which was rarely severe enough to narrow the lumen significantly, and medial fibrosis (Fig. 2B).

Morphometrical analysis. In the morphometric analysis, the ITI of the vessel conduit was calculated as a measure of the degree of preoperative luminal narrowing dependent on intimal thickness (Table III). The mean ITI values for the vessel conduits were 0.37 for the SVs, 0.95 for the RAs, and 1.66 for the ITAs. No patient had $>50 \%$ conduit stenosis.

Risk factor analysis. Table IV shows the incidence rates of selected cardiovascular risk factors associated with vessel conduits. All patients showed risk factors for atherosclerosis, such as age >60 years, arterial hypertension, smoking, DM, obesity and hyperlipidemia. Arterial hypertension was found in $65.38 \%$ of all cases, having an impact on the development of atherosclerotic plaques and fibrointimal hyperplasia. Hyperlipidemia was also present in $65.38 \%$ of all cases. DM was present in $38.46 \%$ of cases; DM is associated with the progression of atherosclerosis in the native vessels and the functional impairment of veins where the level of prostacyclin production is reduced (12). Smoking was a factor present in $34.62 \%$ of all cases; it is an important risk factor for the early and late thrombosis of venous grafts (13). Obesity was less frequently involved, and was present in only $15.38 \%$ of all cases in the study group.

The association between the cumulative number of risk factors and degree of conduit stenosis is shown in Table V. Vascular conduit stenosis of $<25 \%$ (ITI range, 0.18-0.95) was found in 16 patients and $88 \%$ of them $(14 / 16)$ had three or fewer cumulative risk factors. The degree of narrowing was $25-50 \%$ of the vascular conduit (ITI range, 1.02-1.67) in 10 patients and $70 \%$ of them $(7 / 10)$ had more than three cumulative risk factors.

\section{Discussion}

Three types of vascular lesions were identified in the grafts, namely intimal hyperplasia, atherosclerosis and medial fibrosis. 
Table II. Lesion types in the different types of vessel conduit.

\begin{tabular}{lccccc}
\hline & & \multicolumn{3}{c}{ Lesion } \\
\cline { 3 - 5 } Vessel type & Normal & Intimal hyperplasia & Atherosclerosis & Medial fibrosis & Total \\
\hline ITA & 7 & 10 & 2 & 5 & 24 \\
RA & 3 & 4 & 0 & 5 & 12 \\
SV & 5 & 6 & 0 & 7 & 18 \\
Total & 15 & 20 & 2 & 17 & 54 \\
\hline
\end{tabular}

ITA, internal thoracic artery; RA, radial artery; SV, saphenous vein.

Table III. ITI assessment and vessel conduit measurements.

\begin{tabular}{lcccccr}
\hline & & & \multicolumn{3}{c}{ ITI (mean) } \\
\cline { 5 - 6 } Atherosclerosis grade (R) & Degree of intimal thickening & Luminal narrowing & RA & SV & ITA & Total cases \\
\hline Grade 0 & Normal & & 0.00 & 0.00 & 0.00 & 0.00 \\
Grade I $(<0.1)$ & Insignificant & Minimal & 0.00 & 0.00 & 0.00 & 0.00 \\
Grade II $(0.1-1.0)$ & Mild & $<25 \%$ & 0.95 & 0.37 & 0.00 & 16.00 \\
Grade III $(1.0-3.0)$ & Moderate & $25-50 \%$ & 0.00 & 0.00 & 1.66 & 10.00 \\
Grade IV $(>3.0)$ & Severe & $>50 \%$ & 0.00 & 0.00 & 0.00 & 0.00 \\
\hline
\end{tabular}

ITI, intimal thickening index; R, ratio of intimal thickness to medial thickness.

Table IV. Incidence rates of selected risk factors associated with vessel conduits.

\begin{tabular}{lr}
\hline Risk factor & Patients, $\mathrm{n}(\%)$ \\
\hline Age $>60$ years & $16(61.54)$ \\
Smoking & $9(34.62)$ \\
Arterial hypertension & $17(65.38)$ \\
Hyperlipidemia & $17(65.38)$ \\
Diabetes mellitus & $10(38.46)$ \\
Obesity & $4(15.38)$ \\
\hline
\end{tabular}

Mild intimal hyperplasia was observed in the majority of the graft segments taken from the patients undergoing CABG. Intimal hyperplasia occurred more frequently in ITA grafts (10/24 cases, $41.67 \%)$ than in SV grafts (6/18 cases, $33.33 \%)$ and RA grafts (4/12 cases, $33.33 \%)$. Intimal hyperplasia, which was identified in $37.04 \%$ of all vessels, was observed in the majority of the graft samples removed from diabetic patients, who comprised $38.46 \%$ of the study population undergoing CABG. Intimal hyperplasia occurs as a response to physiological stimuli, as the tissue attempts to maintain normal conditions of flow and/or wall tension (14).

Excessive lipoprotein in the plasma may cause atherosclerosis, due to its tendency to accumulate in the hyperplastic intima (15). In the present study, the incidence of hyperlipidemia was $65.38 \%$ (17 cases), and the incidence of atherosclerosis in the ITA was $8.33 \%$ (2/24 cases), whereas no atherosclerosis was identified in the RAs. The prevalence of atherosclerosis in the two arteries may have been underestimated in this study because only the distal ends of arteries were examined, and atherosclerosis is a segmental disease.

When investigated using microscopy, $27.78 \%$ of the specimens were found to be normal, and $31.48 \%$ were mildly to moderately fibrotic. On histopathological evaluation, vascular medial fibrosis of the tunica media was found to be present in all types of vessel grafts, suggesting that fibrosis is a global process that occurs regardless of the involved vessel. Among all cases of medial fibrosis, the SVs accounted for $41.17 \%$ ( 7 cases) , compared with $29.41 \%$ ( 5 cases each) by the RA and ITA, respectively.

Medial vascular fibrosis increases the risk of cardiovascular events by contributing to the stiffening of vessels and reduced vascular compliance (16). In the patients of the present study, the known cardiovascular risk factors hypertension (65.38\%), DM (38.46\%) and age (61.54\%) were present, which may be associated with increased vascular fibrosis. Selvin et al (17) reported that vascular fibrosis is a global process associated with diseases of elevated pulse pressure and aging.

Two indices, ITI and percentage of luminal narrowing, were used for morphometric analysis in the present study. ITI analyses showed that the ITAs had thicker intima than the RAs and SVs. The severity indices reflect the degree of luminal narrowing, and the ITI was significantly greater in the ITAs (1.66) than in the RAs (0.95) and SVs (0.37). These measurements correspond to different degrees of luminal narrowing in the conduit vessels that were used, which were grade III in the 
Table V. Association of the degree of conduct stenosis with the number of cardiovascular risk factors.

\begin{tabular}{|c|c|c|c|c|c|c|c|c|c|}
\hline \multirow[b]{2}{*}{ ITI } & \multirow[b]{2}{*}{ Degree of narrowing } & \multicolumn{7}{|c|}{ Number of risk factors } & \multirow[b]{2}{*}{ Total } \\
\hline & & 7 & 6 & 5 & 4 & 3 & 2 & 1 & \\
\hline Grade IV & $>50 \%$ & - & - & - & - & - & - & - & - \\
\hline Grade III & $25-50 \%$ & 1 & 2 & 2 & 2 & 2 & 1 & - & 10 \\
\hline Grade II & $<25 \%$ & - & - & 1 & 1 & 6 & 5 & 3 & 16 \\
\hline Grade I & Insignificant & - & - & - & - & - & - & - & - \\
\hline Total & & 1 & 2 & 3 & 3 & 8 & 6 & 3 & 26 \\
\hline
\end{tabular}

ITI, intimal thickness index.

A

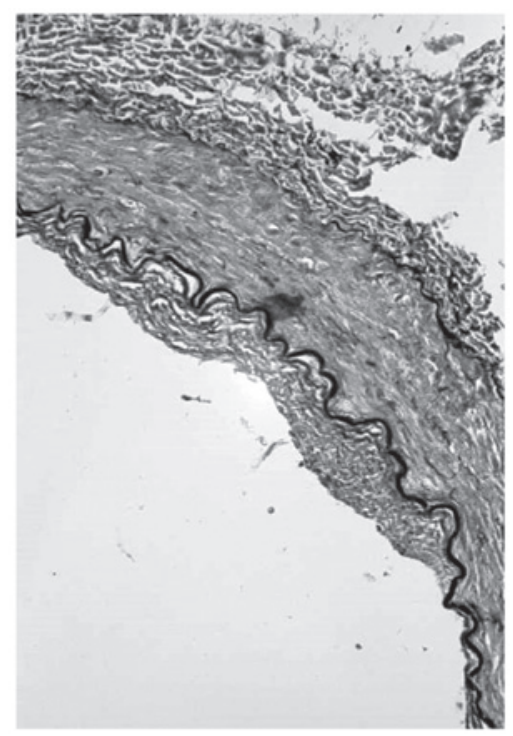

B

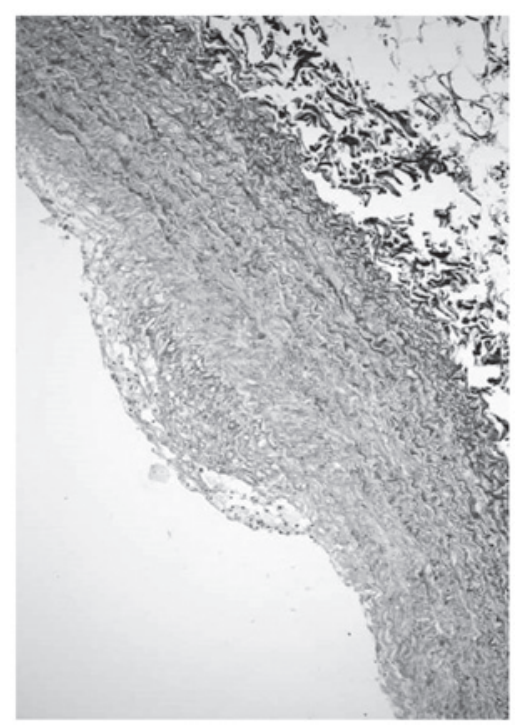

Figure 1. Histological analysis of the internal thoracic artery, showing thickening of the intima. (A) Intimal hyperplasia (elastic Van Gieson staining; magnification, $\mathrm{x} 200$ ) and (B) fatty streaks (hematoxylin and eosin staining; magnification, $\mathrm{x} 200)$.

ITAs and grade II in the RA and VSs. These results indicate that SVs have the least severe pre-existing lesions prior to CABG surgery.
A

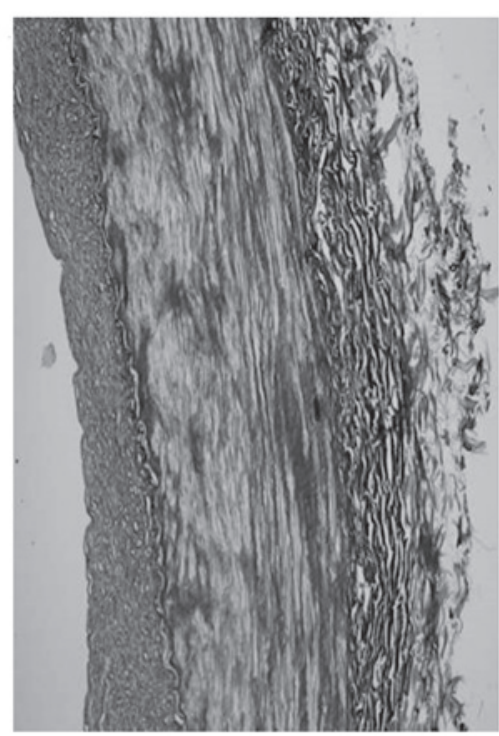

B

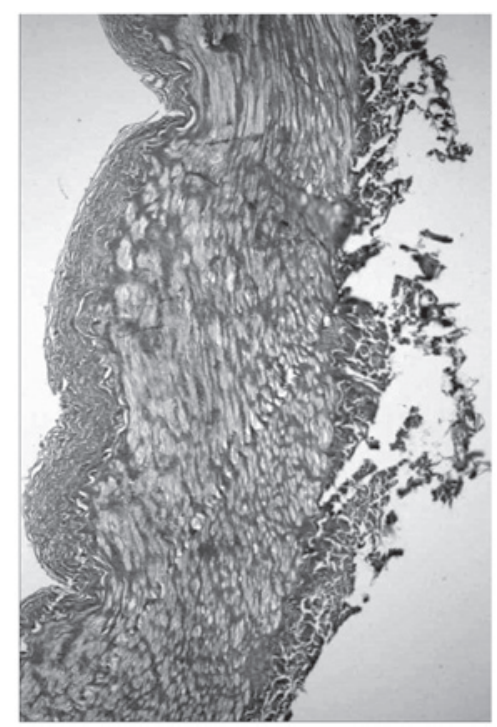

Figure 2. Histological analysis of the (A) radial artery, showing thickening of the intima (Sirius red staining; magnification, x200) and (B) saphenous vein, showing thickening of the intima and media (Sirius red staining, magnification, $\mathrm{x} 200$ ).

The percentage of luminal narrowing indicates the degree of intimal thickening, including atherosclerosis. The area of the intima, rather than the thickness, was measured to allow 
eccentric or irregular lesions to be evaluated more accurately. The percentage of luminal narrowing is considered to be the parameter most useful for comparing intimal thickening in vascular beds of different types (18).

The authors of the present study hypothesized that ITI and IMR may be used to compare the intimal layer thickness in various vascular diseases, which is consistent with a previous study (19). In our previous study we demonstrated that the ITI method was more accurate, since it uses areas, rather than dimensions such as width (20).

There were several limitations to the present study. First, only the changes of ITA, RA and SV histology immediately prior to CABG were evaluated, and secondly, the patients were not clinically followed up, and so it was not possible to correlate $\mathrm{CABG}$ outcome with the histopathological findings.

In conclusion, the present study revealed the presence of preoperative vascular lesions, such as intimal hyperplasia and medial fibrosis, in many of the vessels, which is significant since preoperative lesions are likely to have an effect on the long-term viability of the conduit. Calculation of the ITI provided an indication of the degree of lumen narrowing in the preoperative vascular conduits. The ITI and measurements of intimal and medial areas may serve as reference points in the follow-up assessment of arterial and venous conduit patency. Factors identified in the patients that are likely to be significant predictors of lesion severity (associated with, for example intimal hyperplasia and atherosclerosis) in the CABGs were hyperlipidemia, arterial hypertension, smoking, age and DM.

\section{References}

1. Velazquez EJ, Lee KL, Deja MA, Jain A, Sopko G, Marchenko A, Ali IS, Pohost G, Gradinac S, Abraham WT, et al: Coronary-artery bypass surgery in patients with left ventricular dysfunction. N Engl J Med 364: 1607-1616, 2011.

2. Vassiliades T Jr: Enabling technology for minimally invasive coronary artery bypass grafting. Semin Thorac Cardiovasc Surg 21: 237-244, 2009.

3. Sabik JF III, Lytle BW, Blackstone EH,Houghtaling PL and Cosgrove DM: Comparison of saphenous vein and internal thoracic artery graft patency by coronary system. Ann Thorac Surg 79: 544-551, 2005.

4. Tatoulis J, Buxton BF, Fuller JA, Meswani M, Theodore S, Powar N and Wynne R: Long-term patency of 1108 radial arterial-coronary angiograms over 10 years. Ann Thorac Surg 88: 23-30, 2009.

5. Wijns W, Kolh P, Danchin N, Di Mario C, Falk V, Folliguet T, Garg S, Huber K, James S, Knuuti J, et al; The Task Force on Myocardial Revascularization of the European Society of Cardiology (ESC) and the European Association for Cardio-Thoracic Surgery (EACTS); European Association for Percutaneous Cardiovascular Interventions (EAPCI): Guidelines on myocardial revascularization. Eur Heart J 31: 2501-2555, 2010.
6. Fattouch K, Sampognaro R, Speziale G, Salardino M, Novo G, Caruso M, Novo S and Ruvolo G: Impact of moderate ischemic mitral regurgitation after isolated coronary artery bypass grafting. Ann Thorac Surg 90: 1187-1194, 2010.

7. Beller GA and Ragosta M: Decision making in multivessel coronary disease: The need for physiological lesion assessment. JACC Cardiovasc Interv 3: 315-317, 2010.

8. Leacche M, Balaguer JM and Byrne JG: Intraoperative grafts assessment. Semin Thorac Cardiovasc Surg 21: 207-212, 2009.

9. Turer AT and Hill JA: Pathogenesis of myocardial ischemia-reperfusion injury and rationale for therapy. Am J Cardiol 106: 360-368, 2010.

10. Tarakji KG, Sabik JF III, Bhudia SK, Batizy LH and Blackstone EH: Temporal onset, risk factors and outcomes associated with stroke after coronary artery bypass grafting. JAMA 305: 381-390, 2011.

11. Kobayashi H, Kitamura S, Kawachi K, Morita R, Konishi Y and Tsutsumi M: A pathohistological and biochemical study of arteriosclerosis in the internal thoracic artery, a vessel commonly used as a graft in coronary artery bypass surgery. Surg Today 23: 697-703, 1993.

12. Otsuka F, Yahagi K, Sakakura K and Virmani R: Why is the mammary artery so special and what protects it from atherosclerosis? Ann Cardiothorac Surg 2: 519-526, 2013.

13. Domanski MJ, Borkowf CB, Campeau L, Knatterud GL, White C, Hoogwerf B, Rosenberg Y and Geller NL: Prognostic factors for atherosclerosis progression in saphenous vein grafts: The postcoronary artery bypass graft (Post-CABG) trial. Post-CABG Trial Investigators. J Am Coll Cardiol 36: 1877-1883, 2000.

14. Jones WB, Riley CP, Reeves TJ and Sheffield LT: Natural history of coronary artery disease. Bull N Y Acad Med 48: 1109-1125, 1972.

15. Allon M, Litovsky S, Young CJ, Deierhoi MH, Goodman J, Hanaway M, Lockhart ME and Robbin ML: Correlation of pre-existing vascular pathology with arteriovenous graft outcomes in hemodialysis patients. Am J Kidney Dis 62: $1122-1129,2013$

16. Sulikowski T, Tejchman K, Ziętek Z, Urasińska E, Domański L, Sieńko J, Romanowski M, Safranow K, Zukowski M, Ciechanowicz A, et al: Histopathologic evaluation of pretransplantation biopsy as a factor influencing graft function after kidney transplantation in 3-year observation. Transplant Proc 42: 3375-3381, 2010.

17. Selvin E, Najjar SS, Cornish TC and Halushka MK: A comprehensive histopathological evaluation of vascular medial fibrosis: Insights into the pathophysiology of arterial stiffening. Atherosclerosis 208: 69-74, 2010.

18. Thiene G, Miazzi P, Valsecchi M, Valente M, Bortolotti U, Casarotto D and Gallucci V: Histological survey of the saphenous vein before its use as autologous aortocoronary bypass graft. Thorax 35: 519-522, 1980.

19. Ruengsakulrach P, Sinclair R, Komeda M, Raman J, Gordon I and Buxton B: Comparative histopathology of radial artery versus internal thoracic artery and risk factors for development of intimal hyperplasia and atherosclerosis. Circulation 100 (19 Suppl): II139-II144, 1999.

20. Tinică G, Luca C, Dumitras EA and Butcovan D: Pre-operative graft assessment before their use in aortocoronary by-pass surgery. In: The $10^{\text {th }}$ Congress of the Romanian Society of Cardiovascular Surgery. International Hotel, Iasi pp17, 2014. 